\title{
Baleen-mimicking virtual filter for rapid detection of pathogenic bacteria in water using magnetic nanoparticle chains and a Halbach ring
}

\author{
Hyeonjeong Lee, Hyunsoo Han and Sangmin Jeon* \\ Department of Chemical Engineering, Pohang University of Science and Technology \\ (POSTECH), Pohang, Gyeongbuk, Republic of Korea \\ *Corresponding author.E-mail: jeons@postech.ac.kr
}

(a)

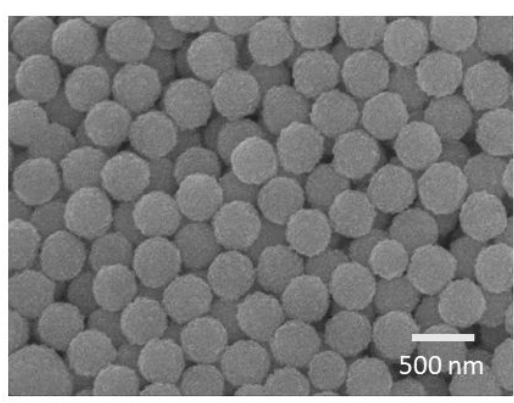

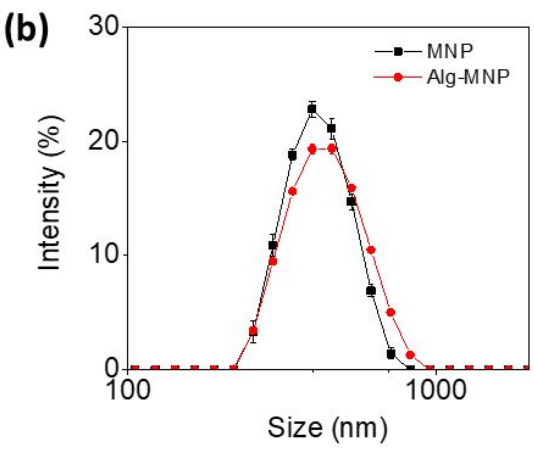

Figure S1. (a) SEM image of Alg-MNPs and (b) hydrodynamic sizes of MNPs and Alg-MNPs measured by dynamic light scattering. 

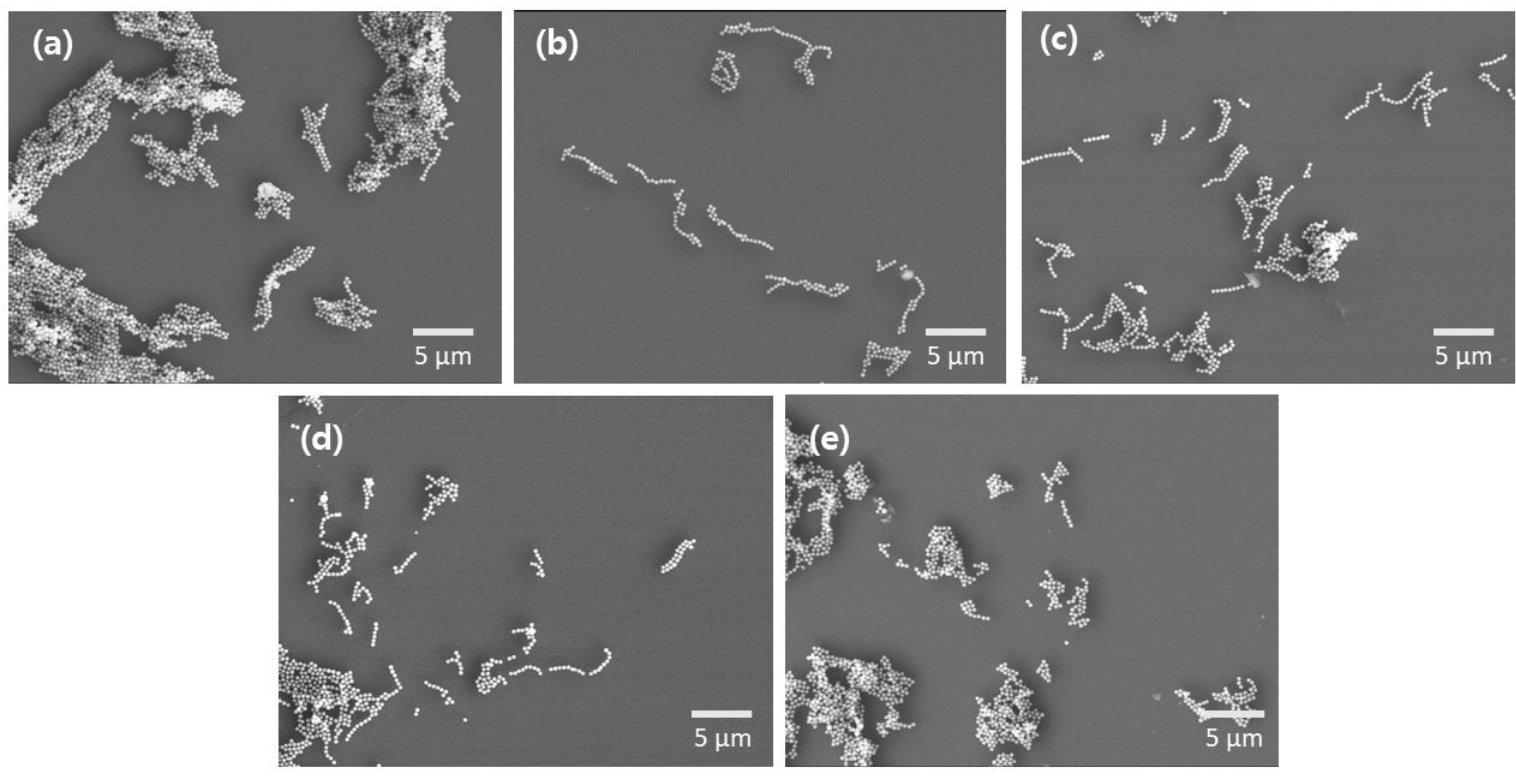

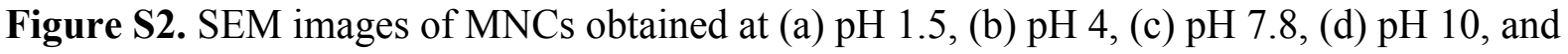
(e) $\mathrm{pH} 13.5$ of citric acid.

(a)

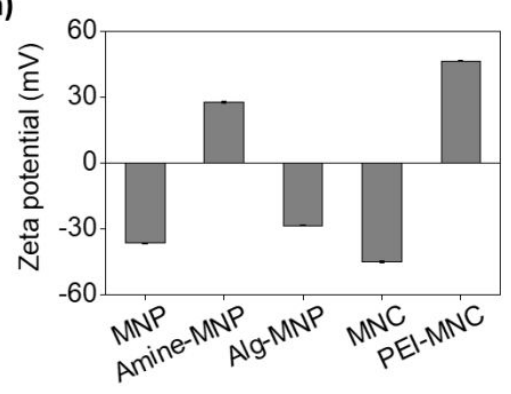

(b)

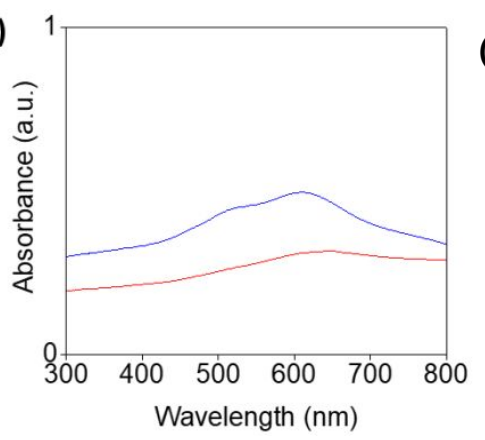

(c)

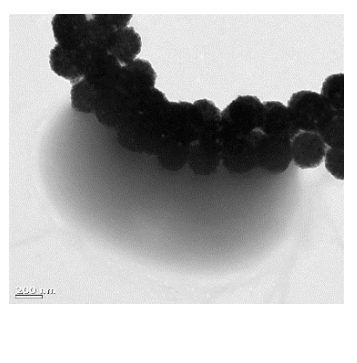

Figure S3. (a) Changes in zeta potential of MNPs and MNCs before and after various coatings. (b) UV-Vis absorption spectra of MNPs (blue) and MNCs (red). (c) TEM image of a PEIMNC-bound E. coli bacterium. The scale bar corresponds to $200 \mathrm{~nm}$. 


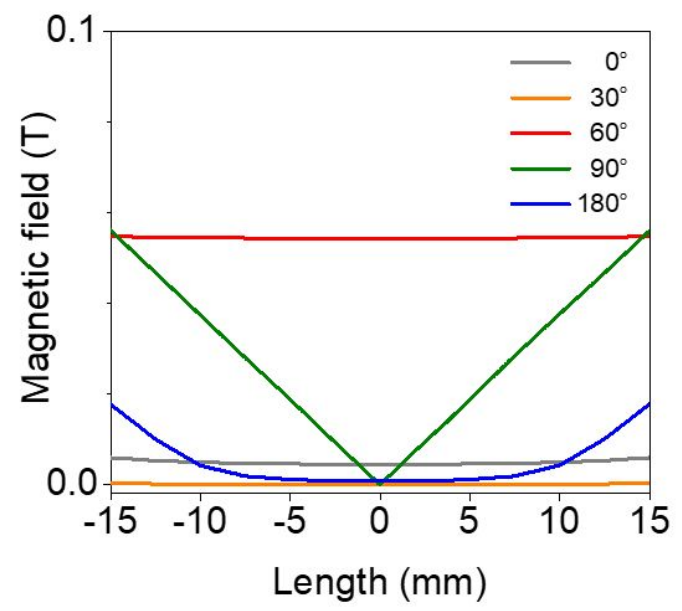

Figure S4. Magnetic field strengths in the radial direction through the center of ring magnet arrays with various $\Delta \varphi$.

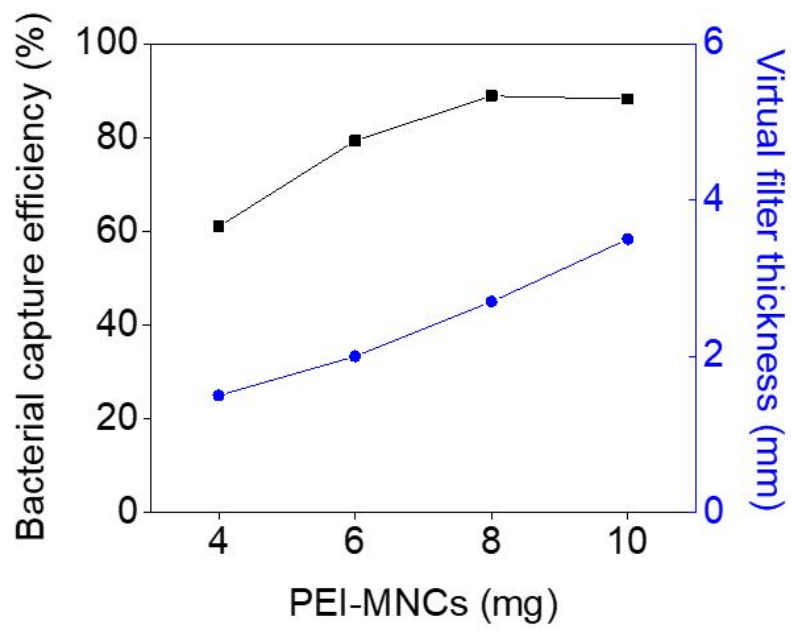

Figure S5. Variations in the bacterial capture efficiencies and thickness of the virtual filters as a function of the amount of PEI-MNC. The flow rate was fixed at $5 \mathrm{~mL} / \mathrm{min}$. As the amount of PEI-MNC increased, the virtual filter thickness increased linearly, but the bacterial capture efficiency was saturated when the amount of PEI-MNC was $8 \mathrm{mg}$ or more. 


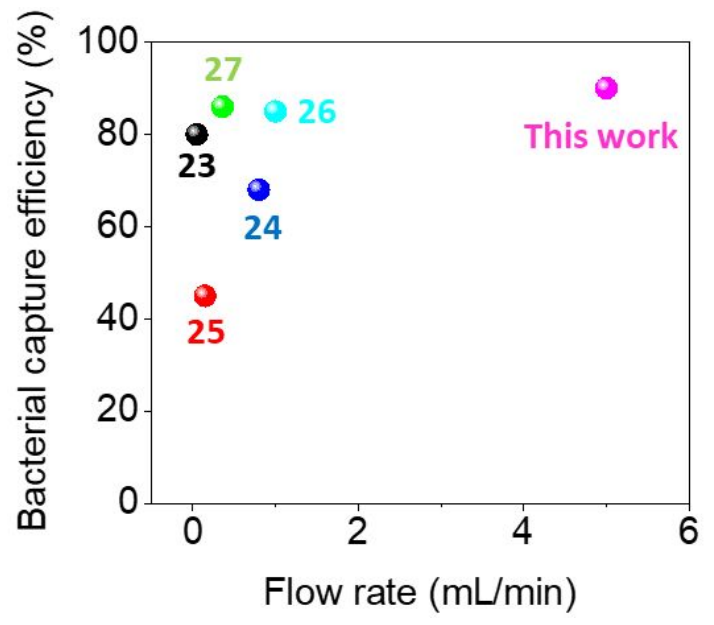

Figure S6. Comparison of various virtual filters for bacterial capture efficiency. The numbers in the graph correspond to the reference numbers in the manuscript. 